IMA Journal of Applied Mathematics (2017) 82, 849-863

doi:10.1093/imamat/hxx017

Advance Access Publication on 3 July 2017

\title{
Limit cycles bifurcating from discontinuous centres
}

\author{
Denis de CARvalho BRaga \\ Instituto de Matemática e Computação, Universidade Federal de Itajubá, Avenida BPS 1303, \\ Pinheirinho, CEP 37.500-903 Itajubá, MG, Brazil \\ braga@unifei.edu.br \\ Tiago Carvalho \\ Departamento de Matemática, Universidade Estadual Paulista, Rua Eng. Luiz Edmundo Carrijo \\ Coube, 14-01, CEP 17.033-360 Bauru, SP, Brazil \\ tcarvalho@fc.unesp.br \\ AND \\ Luis Fernando Mello* \\ Instituto de Matemática e Computação, Universidade Federal de Itajubá, Avenida BPS 1303, \\ Pinheirinho, CEP 37.500-903 Itajubá, MG, Brazil \\ *Corresponding author:1fmelo@unifei.edu.br
}

[Received on 15 February 2016; revised on 16 November 2016; accepted on 24 May 2017]

\begin{abstract}
In this article, we study limit cycles in discontinuous piecewise linear vector fields in $\mathbb{R}^{2}$ and $\mathbb{R}^{3}$. More precisely, we address the problem of understanding the dynamics around a degenerated two-fold singularity in $\mathbb{R}^{2}$ and $\mathbb{R}^{3}$, where it is also called T-singularity, which behave as discontinuous centres after suitable perturbations of the separation boundaries. We prove that, in both systems, it is possible to obtain $k$ hyperbolic limit cycles bifurcating from these discontinuous centres, for any positive integer $k$. The same holds if $k$ is infinity.
\end{abstract}

Keywords: bifurcation; limit cycle; non-smooth vector field; T-singularity; two-fold singularity.

\section{Introduction}

One of the most challenging problems in the qualitative theory of ordinary differential equations in 2D and 3D is to study the existence, maximum number and distribution of limit cycles. This problem is related with the second part of the 16th Hilbert problem (see Hilbert, 1902) when the differential equations are polynomial. In this article, we study limit cycles in discontinuous piecewise linear vector fields (PWLVF) in $\mathbb{R}^{2}$ and $\mathbb{R}^{3}$. More precisely, we study limit cycles bifurcating from discontinuous centres defined by two-fold singularities in $\mathbb{R}^{2}$ and $\mathbb{R}^{3}$.

On the one hand, the study of oscillations and periodic phenomena in physical systems has attracted great attention due to the interpretations assigned to them. When these physical systems are modelled by ordinary differential equations, the mathematical subject underlying to an oscillation is a periodic orbit. In particular, these oscillations and periodic phenomena can be described mathematically in terms of limit cycles which are periodic orbits isolated in the set of all periodic orbits. The study of limit cycles goes back to the works of Poincaré, Liénard, van der Pol, Andronov, among others. See the article Ginoux \& Petitgirard (2010) for historical comments and references and the book Andronov et al. (1966) considered the landmark for the mathematical study of oscillations.

() The authors 2017. Published by Oxford University Press on behalf of the Institute of Mathematics and its Applications. All rights reserved. 
On the other hand, piecewise smooth vector fields in $\mathbb{R}^{2}$ or $\mathbb{R}^{3}$ have been attracted great attention in recent years. See the books Andronov et al. (1966), Filippov (1988), Llibre \& Teruel (2014) and references therein. In particular, great emphasis has been given to piecewise linear differential systems with two or more zones. Piecewise linear differential systems with two zones in $\mathbb{R}^{n}$ are generally defined by

$$
X^{\prime}=\left\{\begin{array}{l}
F^{-}(X)=A^{-} X+B^{-}, \mathscr{H}(X) \leq 0, \\
F^{+}(X)=A^{+} X+B^{+}, \mathscr{H}(X) \geq 0,
\end{array}\right.
$$

where the prime denotes derivative with respect to the independent variable $t$, called here the time, $X \in \mathbb{R}^{n}, A^{ \pm}$are $n \times n$ real matrices, $B^{ \pm}$are $n \times 1$ real matrices, the function $\mathscr{H}: \mathbb{R}^{n} \rightarrow \mathbb{R}$ is at least continuous and the set $\Sigma=\mathscr{H}^{-1}(0)$ divides $\mathbb{R}^{n}$ in two unbounded components (zones) $\Sigma^{+}$and $\Sigma^{-}$ where $\mathscr{H}$ is positive and negative, respectively. Thus $\mathbb{R}^{n}=\Sigma^{+} \cup \Sigma \cup \Sigma^{-}$. We denote (1.1) simply by $Z=\left(F^{-}, F^{+}, \mathscr{H}\right)$.

Since the seminal work in Andronov et al. (1966), a lot of articles were published mainly about questions like the existence, number, stability and distribution of limit cycles. These studies were developed taking into account aspects like the number and stability of equilibria as well as their locations with respect to the separation boundary $\Sigma$ and the behaviour of the linear vector fields on $\Sigma$. Usually, the points of discontinuity on the separation boundary are classified as sewing or crossing, sliding, escaping or tangency points. See Section 2.

The separation boundary $\Sigma$ between the two zones plays an important role in planar discontinuous piecewise linear differential systems. In the article by Braga \& Mello (2014) was exhibited an example of such a system with seven hyperbolic limit cycles having $\Sigma$ as a polygonal curve. In the article Braga $\&$ Mello (2015), the authors proved the existence of a class of discontinuous piecewise linear differential systems with two zones in the plane separated by a polygonal curve $\Sigma$ having exactly $n$ hyperbolic limit cycles, for any given positive integer $n$.

Examples of piecewise linear differential systems with two zones separated by a straight line in the plane with three crossing limit cycles are known. See the articles Braga \& Mello (2013), Freire et al. (2014) and Llibre \& Ponce (2012). Nevertheless, it is not proved that three is the maximum number of crossing limit cycles of those systems.

Consider the following piecewise linear differential system in the plane

$$
X^{\prime}= \begin{cases}F^{-}(x, y)=(1,2 x), & y \leq 0, \\ F^{+}(x, y)=(-1,2 x), & y \geq 0 .\end{cases}
$$

In (1.2), the separation boundary is given by $\Sigma=\mathscr{H}^{-1}(0)$ where $\mathscr{H}(x, y)=y$ and all the points on $\Sigma$ are of crossing type, except the origin which is an invisible tangent point (see Section 2 for precise definitions) for both linear vector fields $F^{+}$and $F^{-}$. It is simple to see that all the orbits of (1.2) are crossing periodic orbits, except those by the origin. The phase portrait of (1.2) is illustrated in Fig. 1. We call it a non-degenerate $\Sigma$-centre.

In Buzzi et al. (2014), nonlinear perturbations of the linear vector fields $F^{-}$and $F^{+}$in (1.2) were studied, keeping the separation line $\Sigma$ fixed, in order to obtain an arbitrary number of limit cycles in the nonlinear perturbed system. In this article, we obtain the same conclusions of Buzzi et al. (2014) but by a different process: we perturb the separation curve $\Sigma$ keeping the linear vector fields $F^{-}$and $F^{+}$in (1.2) unchanged. More precisely, we have the following theorem. 


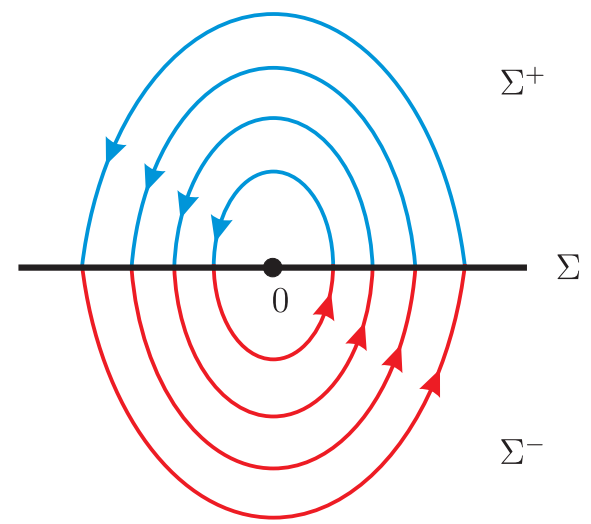

FIG. 1. Phase portrait of system (1.2). Non-degenerate $\Sigma$-centre.

THEOREM 1.1 Consider the piecewise linear differential system in the plane $Z=\left(F^{-}, F^{+}, \mathscr{H}\right)$ defined in (1.2). Given a positive integer $k$, there is a smooth perturbation of the function $\mathscr{H}$, denoted by $\mathscr{H}_{k}$, such that the piecewise linear differential system in the plane $Z_{k}=\left(F^{-}, F^{+}, \mathscr{H}_{k}\right)$ has $k$ hyperbolic limit cycles. The same holds if $k=\infty$.

Now consider the following piecewise linear differential system in $\mathbb{R}^{3}$

$$
X^{\prime}= \begin{cases}F^{-}(x, y, z)=(1,-1, x), & z \leq 0 \\ F^{+}(x, y, z)=(-1-(x+y), 1-(x+y),-y), & z \geq 0\end{cases}
$$

In (1.3), the separation boundary is given by $\Sigma=\mathscr{L}^{-1}(0)$ where $\mathscr{L}(x, y, z)=z$.

As we shall make clear in Section 4, system (1.3) is structurally unstable and their flow restrict to the invariant plane $x+y=0$ is like in Fig. 1, that is all the orbits in the invariant plane $x+y=0$ are crossing periodic orbits, except those by the origin.

Nonlinear perturbations of the linear vector fields $F^{-}$and $F^{+}$in (1.3), keeping the separation plane $\Sigma$ fixed, were studied in Carvalho \& Teixeira (2015). The authors proved the existence of an arbitrary number of limit cycles in the nonlinear perturbed system. In this article, by perturbing the separation plane $\Sigma$ and keeping the linear vector fields $F^{-}$and $F^{+}$in (1.3) unchanged, we obtain the same conclusions of Carvalho \& Teixeira (2015). More precisely, we have the following theorem.

THeOREM 1.2 Let $Z=\left(F^{-}, F^{+}, \mathscr{L}\right)$ be the piecewise linear differential system in $\mathbb{R}^{3}$ defined in (1.3). Given a positive integer $k$, there is a smooth perturbation of the function $\mathscr{L}$, denoted by $\mathscr{L}_{k}$, such that the piecewise linear differential system in $\mathbb{R}^{3} Z_{k}=\left(F^{-}, F^{+}, \mathscr{L}_{k}\right)$ has $k$ hyperbolic limit cycles in the plane $x+y=0$. The same holds if $k=\infty$.

In our opinion, the procedures applied to obtain limit cycles bifurcating from two-fold singularities in Theorems 1.1 and 1.2 are more simple and more intuitive than the ones in Buzzi et al. (2014) and Carvalho \& Teixeira (2015). 
The article is organized as follows. We introduce terminologies, some definitions and the basic theory about piecewise linear differential systems in Section 2. In Section 3, we prove Theorem 1.1. Theorem 1.2 is proved in Section 4.

\section{Preliminaries}

Our interest in this article is to study qualitative aspects of PWLVF in $\mathbb{R}^{2}$ and $\mathbb{R}^{3}$ defined by $Z=$ $\left(F^{-}, F^{+}, \mathscr{H}\right)$. See equation (1.1).

The basic results of differential equations in this context were first stated in the seminal work (Filippov, 1988) that we summarize next. Indeed, consider the Lie derivatives

$$
F^{ \pm} \mathscr{H}(p)=\left\langle\nabla \mathscr{H}(p), F^{ \pm}(p)\right\rangle
$$

and

$$
\left(F^{ \pm}\right)^{i} \mathscr{H}(p)=\left\langle\nabla\left(\left(F^{ \pm}\right)^{i-1} \mathscr{H}\right)(p), F^{ \pm}(p)\right\rangle, \quad i \geq 2,
$$

where $\langle.,$.$\rangle is the usual inner product in \mathbb{R}^{n}, n=2,3$.

We distinguish the following subsets of the discontinuity set $\Sigma$ :

(i) Crossing set defined by

$$
\Sigma^{c}=\left\{p \in \Sigma \mid\left(F^{+} \mathscr{H}(p)\right)\left(F^{-} \mathscr{H}(p)\right)>0\right\} .
$$

Moreover, we denote

$$
\Sigma^{c+}=\left\{p \in \Sigma \mid F^{+} \mathscr{H}(p)>0, F^{-} \mathscr{H}(p)>0\right\}
$$

and

$$
\Sigma^{c-}=\left\{p \in \Sigma \mid F^{+} \mathscr{H}(p)<0, F^{-} \mathscr{H}(p)<0\right\} .
$$

(ii) Sliding set defined by

$$
\Sigma^{s}=\left\{p \in \Sigma \mid F^{+} \mathscr{H}(p)<0, F^{-} \mathscr{H}(p)>0\right\}
$$

(iii) Escaping set defined by

$$
\Sigma^{e}=\left\{p \in \Sigma \mid F^{+} \mathscr{H}(p)>0, F^{-} \mathscr{H}(p)<0\right\} .
$$


When $q \in \Sigma^{s} \cup \Sigma^{e}$, following the Filippov's convention, the sliding vector field associated to $Z=\left(F^{-}, F^{+}, \mathscr{H}\right)$ is the vector field $\widehat{Z}^{s}$ tangent to $\Sigma^{s} \cup \Sigma^{e}$ and expressed as

$$
\begin{aligned}
\widehat{Z}^{s} & =\frac{1}{\left\langle\left(F^{-}-F^{+}\right), \nabla \mathscr{H}\right\rangle}\left[\left\langle F^{-}, \nabla \mathscr{H}\right\rangle F^{+}-\left\langle F^{+}, \nabla \mathscr{H}\right\rangle F^{-}\right] \\
& =\frac{\left(F^{-} \mathscr{H}\right) F^{+}-\left(F^{+} \mathscr{H}\right) F^{-}}{F^{-} \mathscr{H}-F^{+} \mathscr{H}} .
\end{aligned}
$$

Associated to (2.1), there exists the normalized sliding vector field

$$
Z^{s}=\left(F^{-} \mathscr{H}\right) F^{+}-\left(F^{+} \mathscr{H}\right) F^{-} .
$$

Note that $Z^{s}$ can be $\mathscr{C}^{\infty}$-extended to the boundary of $\Sigma^{s}$. The $f$ low of $Z=\left(F^{-}, F^{+}, \mathscr{H}\right)$ is obtained by the concatenation of the flows of $F^{-}, F^{+}$and $Z^{s}$.

Remark 2.1 If $q \in \Sigma^{s}$ then $F^{-} \mathscr{H}-F^{+} \mathscr{H}>0$, therefore $\widehat{Z}^{s}$ and $Z^{s}$ are topologically equivalent in $\Sigma^{s}$ since they have the same orientation and can be $\mathscr{C}^{\infty}$-extended to the closure of $\Sigma^{s}$. If $q \in \Sigma^{e}$ then $\widehat{Z}^{s}$ and $Z^{s}$ have opposite orientations since $F^{-} \mathscr{H}-F^{+} \mathscr{H}<0$.

In this context, a richer dynamics occurs on those points $q \in \Sigma^{s} \cup \Sigma^{e}$ such that $Z^{s}(q)=0$, called pseudo-equilibria of $Z$, and those points $p \in \Sigma$ such that $\left(F^{+} \mathscr{H}(p)\right)\left(F^{-} \mathscr{H}(p)\right)=0$, called tangential singularities of $Z$, where the trajectory through $p$ is tangent to $\Sigma$.

We say that a point $p \in \Sigma$ is a fold point of $F^{+}$if $F^{+} \mathscr{H}(p)=0$ but $\left(F^{+}\right)^{2} \mathscr{H}(p) \neq 0$. Moreover, $p \in \Sigma$ is a visible (respectively, invisible) fold point of $F^{+}$if $F^{+} \mathscr{H}(p)=0$ and $\left(F^{+}\right)^{2} \mathscr{H}(p)>0$ (respectively, $\left(F^{+}\right)^{2} \mathscr{H}(p)<0$ ). The same definitions for $F^{-}$reversing the inequalities.

We say that $q \in \Sigma$ is a non-degenerate $\Sigma$-centre of $Z=\left(F^{-}, F^{+}, \mathscr{H}\right)$ if $q$ is an invisible fold point for both $F^{-}$and $F^{+}$and there is an invariant plane filled up with a one parameter family of closed orbits of $Z=\left(F^{-}, F^{+}, \mathscr{H}\right)$ in such a way that the orientation is preserved (see Fig. 1).

Denote by $S_{F^{+}}$(respectively, $S_{F^{-}}$), the set of all tangential singularities of $F^{+}$(respectively, $F^{-}$). The subset of $\Sigma$ at which $S_{F^{+}}$and $S_{F^{-}}$intersect is called a two-fold singularity. This singularity is a prototypical model in the generic classification of singularities in PWLVF. As pointed out in Colombo \& Jeffrey (2011), a 3D two-fold singularity is an important organizing centre because it brings together all of the basic forms of dynamics possible in a PWLVF.

There are many distinct topological types of 3D two-fold singularities and the most interesting of them is the so called T-singularity. We say that $p^{*}$ is a T-singularity (or Teixeira-singularity or invisible two-fold singularity) of $Z=\left(F^{-}, F^{+}, \mathscr{H}\right)$ defined in $\mathbb{R}^{3}$, if $p^{*}$ is an invisible fold point of both $F^{-}$and $F^{+}$and the intersection of $S_{F^{+}}$and $S_{F^{-}}$is transversal at $p^{*}$ (see Fig. 2). It is easy to check that the origin of the system (1.3) is a T-singularity. The interested reader can see more details about the T-singularity in Colombo \& Jeffrey (2009, 2011), di Bernardo et al. (2010), Jacquemard et al. (2012), Jacquemard et al. (2013) and Teixeira (1990).

Suppose $p^{*}$ is a T-singularity of $Z=\left(F^{-}, F^{+}, \mathscr{H}\right)$ and consider $p \in \Sigma^{c+}$. It is easy to see that there exists a time $t^{+}(p)>0$, called $F^{+}$-fly time, such that the forward trajectory of $F^{+}$passing through $p$ at $t=0$ returns to $\Sigma$ after $t^{+}(p)$. We define the half return map associated to $F^{+}$by $\varphi_{F^{+}}(p)=\phi_{F^{+}}\left(t^{+}(p), p\right)=p_{1} \in \Sigma$, where $\phi_{F^{+}}$is the flow of $F^{+}$. When $p_{1} \in \Sigma^{c-}$, let $t^{-}\left(p_{1}\right)>0$ be the $F^{-}$-fly time of the trajectory of $F^{-}$passing through $p_{1}$. Define the half return map associated to $F^{-}$ by $\varphi_{F^{-}}\left(p_{1}\right)=\phi_{F^{-}}\left(t^{-}\left(p_{1}\right), p_{1}\right) \in \Sigma$, where $\phi_{F^{-}}$is the flow of $F^{-}$. The $\mathscr{C}^{\infty}$ involution $\varphi_{F^{+}}$(respectively, 


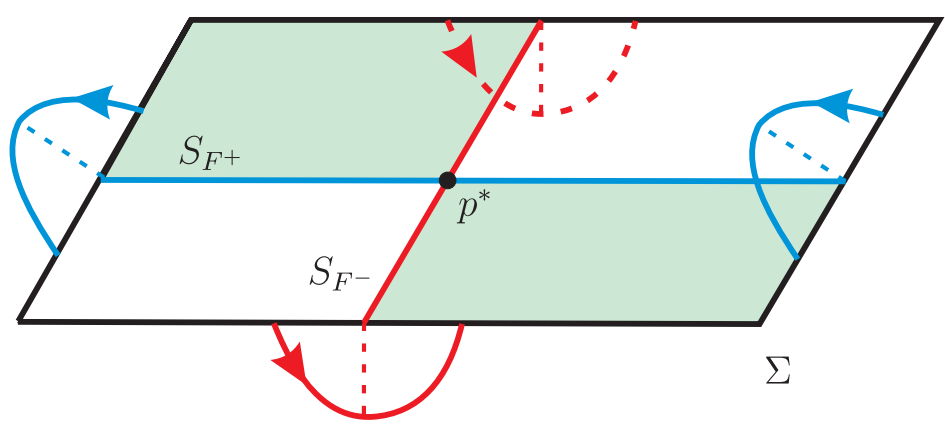

FIG. 2. T-singularity.

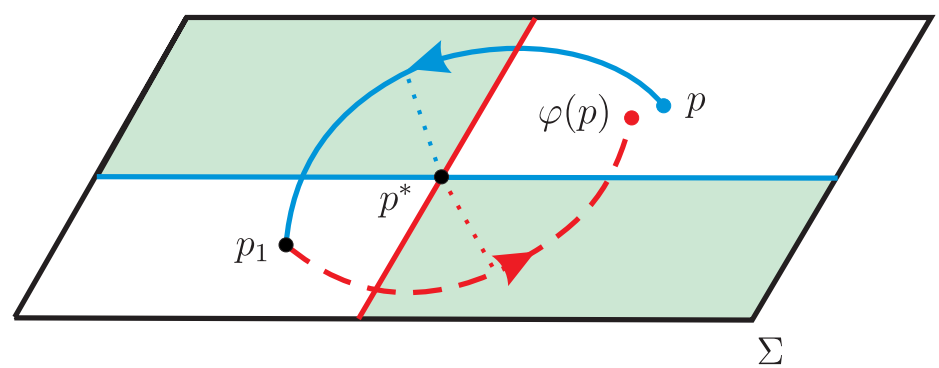

FIG. 3. First return map.

$\left.\varphi_{F^{-}}\right)$is such that $\operatorname{Fix}\left(\varphi_{F^{+}}\right)=S_{F^{+}}$(respectively, $\left.\operatorname{Fix}\left(\varphi_{F^{-}}\right)=S_{F^{-}}\right)$. The first return map associated to $Z=\left(F^{-}, F^{+}, \mathscr{H}\right)$ is defined by the composition of these involutions, that is

$$
\varphi(p)=\varphi_{F^{-}} \circ \varphi_{F^{+}}(p)=\phi_{F^{-}}\left(t^{-}\left(p_{1}\right), \phi_{F^{+}}\left(t^{+}(p), p\right)\right)
$$

or the reverse, applying first the flow of $F^{-}$and after the flow of $F^{+}$. See Fig. 3 and more details in Teixeira (2009).

\section{The planar case}

In this section, we analyse bifurcations of limit cycles from the two-fold singularity given by (1.2) when we perturb the separation line $\Sigma$.

\subsection{Proof of Theorem 1.1}

Let $\mathscr{M}$ be the class of all functions $\mathscr{H}_{k}: \mathbb{R}^{2} \rightarrow \mathbb{R}$ defined by

$$
\mathscr{H}_{k}(x, y)=y-h_{k}(x)
$$

where $(x, y) \in \mathbb{R}^{2}$ and the function $h_{k}$ satisfies the following assumptions:

$$
h_{k} \in \mathscr{C}^{r}(\mathbb{R}, \mathbb{R}) \text {, with } r \in \mathbb{Z}, r \geq 1 \text { or } r=\infty \text {. }
$$


(H2) $h_{k}(x)=0$, if $x \leq 0$ and has a finite $(k \in \mathbb{Z}, k \geq 1)$ or countably infinite $(k=\infty)$ number of zeros for $x>0$. Moreover, if $x_{0}>0$ is a zero, then $h_{k}^{\prime}\left(x_{0}\right) \neq 0$. Here the prime denotes the derivative of $h_{k}$ with respect to $x$.

(H3)

$F^{+} \mathscr{H}_{k}(x, y)>0$ and $F^{-} \mathscr{H}_{k}(x, y)>0$, for all $x>0$.

Since $F^{+} \mathscr{H}_{k}(x, y)=h_{k}^{\prime}(x)+2 x$ and $F^{-} \mathscr{H}_{k}(x, y)=-h_{k}^{\prime}(x)+2 x$, the hypothesis (H3) means that

$$
\left(\frac{h_{k}^{\prime}(x)}{2}\right)^{2}<x^{2}, \quad \forall x>0
$$

Moreover, all the points on $\Sigma_{k}=\left\{(x, y) \in \mathbb{R}^{2}: \mathscr{H}_{k}(x, y)=0\right\}$ are of crossing type.

The set $\mathscr{M}$ is not empty as will be shown later. The hypothesis (H3) will also allow us to build a displacement function

$$
\delta_{k}:(0, \infty) \rightarrow \mathbb{R}
$$

of the system

$$
X^{\prime}= \begin{cases}F^{-}(X)=(1,2 x), & \mathscr{H}_{k}(X) \leq 0, \\ F^{+}(X)=(-1,2 x), & \mathscr{H}_{k}(X) \geq 0\end{cases}
$$

such that each zero of the function $\delta_{k}$ will correspond to a crossing hyperbolic limit cycle by (H2).

Let $\phi_{F^{-}}: \mathbb{R} \times \mathbb{R}^{2} \rightarrow \mathbb{R}^{2}$ and $\phi_{F^{+}}: \mathbb{R} \times \mathbb{R}^{2} \rightarrow \mathbb{R}^{2}$ be the flows associated with the differential equations $X^{\prime}=F^{-}(X)$ and $X^{\prime}=F^{+}(X)$, respectively, where

$$
\begin{aligned}
& \phi_{F^{-}}(t, X)=\left(x^{-}(t, X), y^{-}(t, X)\right)=\left(t+x, t^{2}+2 t x+y\right), \\
& \phi_{F^{+}}(t, X)=\left(x^{+}(t, X), y^{+}(t, X)\right)=\left(-t+x,-t^{2}+2 t x+y\right) .
\end{aligned}
$$

Taking a point $p=\left(x, h_{k}(x)\right) \in \Sigma_{k}^{+}$, there exists a unique $F^{+}$-fly time $t^{+}(p)>0$ such that $\phi_{F^{+}}\left(t^{+}(p), p\right) \in \Sigma_{k}^{-}$, where

$$
\Sigma_{k}^{+}=\left\{X=(x, y) \in \Sigma_{k}: x>0\right\}, \quad \Sigma_{k}^{-}=\left\{X=(x, y) \in \Sigma_{k}: x<0\right\} .
$$

In a similar way, there exists a unique $F^{-}-$fly time $t^{-}(p)>0$, such that $\phi_{F^{-}}\left(-t^{-}(p), p\right) \in \Sigma_{k}^{-}$. The times $t^{+}(p)$ and $t^{-}(p)$ are solutions of $y^{+}\left(t^{+}, p\right)=0$ and $y^{-}\left(-t^{-}, p\right)=0$, respectively, and are given by

$$
t^{+}(p)=x+\sqrt{x^{2}+h_{k}(x)}, \quad t^{-}(p)=x+\sqrt{x^{2}-h_{k}(x)} .
$$

Note that, by hypothesis (H3), the previous times are well defined as functions of $x$, since

$$
\left|h_{k}(x)\right|=\left|\int_{0}^{x} h_{k}^{\prime}(u) \mathrm{d} u\right| \leq \int_{0}^{x}\left|h_{k}^{\prime}(u)\right| \mathrm{d} u<\int_{0}^{x} 2 u \mathrm{~d} u=x^{2},
$$

for each $x>0$. 
Therefore, by (3.3) and (3.4), the displacement function (3.1) is given by

$$
\begin{aligned}
\delta_{k}(x) & =x^{-}\left(-t^{-}(p), p\right)-x^{+}\left(t^{+}(p), p\right) \\
& =\sqrt{x^{2}+h_{k}(x)}-\sqrt{x^{2}-h_{k}(x)}, x \in(0, \infty) .
\end{aligned}
$$

If $x_{0}>0$ is a zero of the function $h_{k}$, then $\delta_{k}\left(x_{0}\right)=0$ and since

$$
\delta_{k}^{\prime}(x)=\frac{1}{2}\left(\frac{h_{k}^{\prime}(x)-2 x}{\sqrt{x^{2}-h_{k}(x)}}+\frac{h_{k}^{\prime}(x)+2 x}{\sqrt{x^{2}+h_{k}(x)}}\right),
$$

it follows that $\delta_{k}^{\prime}\left(x_{0}\right)=h_{k}^{\prime}\left(x_{0}\right) / x_{0} \neq 0$ by hypothesis $(\mathrm{H} 2)$. Thus, $x_{0}>0$ corresponds to a crossing hyperbolic limit cycle of (3.2) which is stable when $h_{k}^{\prime}\left(x_{0}\right)<0$ (see the definition of $\delta_{k}$ in (3.5)) and unstable when $h_{k}^{\prime}\left(x_{0}\right)>0$.

In short, Theorem 1.1 is proved.

\subsection{Some examples}

Consider the set $\mathscr{M}_{\varepsilon}$ of functions $\mathscr{H}_{\varepsilon, k}: \mathbb{R}^{2} \rightarrow \mathbb{R}$ such that $\mathscr{H}_{\varepsilon, k}(X)=y-h_{k}(x)$, where $h_{k}(x)=\varepsilon g_{k}(x)$, $\varepsilon$ is a positive real number and $g_{k}$ has the following properties:

(P1) $g_{k} \in \mathscr{C}^{r}(\mathbb{R}, \mathbb{R}), r \in \mathbb{Z}, r \geq 2$ or $r=\infty$;

(P2) $g_{k}$ has a finite or countably infinite number of zeros for $x>0$. Moreover, if $x_{0}>0$ is a zero, then $g_{k}^{\prime}\left(x_{0}\right) \neq 0$;

(P3) $g_{k}(x)=0$, if $x<0, g_{k}(0)=g_{k}^{\prime}(0)=0$ and $g_{k}^{\prime}(x) \rightarrow L \in \mathbb{R}$ when $x \rightarrow+\infty$.

Applying Taylor's theorem and using the properties (P1) and (P3), there exist positive real numbers $\delta$ and $M$, with $\delta$ sufficiently small, such that

$$
\left(\frac{g_{k}^{\prime}(x)}{2}\right)^{2} \leq M x^{2}
$$

for $x \in[0, \delta]$. So also there exists $\varepsilon>0$ sufficiently small for which

$$
\left(\frac{h_{k}^{\prime}(x)}{2}\right)^{2}=\varepsilon^{2}\left(\frac{g_{k}^{\prime}(x)}{2}\right)^{2} \leq \varepsilon^{2} M x^{2}<x^{2},
$$

for all $x>0$, since the function $g_{k}^{\prime}$ is bounded for $x \geq 0$. Indeed, by the properties (P1) and (P3), given $\eta=1$ there exists $x_{1}>0$ such that ||$g_{k}^{\prime}(x)|-| L|| \leq\left|g_{k}^{\prime}(x)-L\right|<1$ for all $x>x_{1}$, that is, $\left|g_{k}^{\prime}(x)\right|<1+|L|$ when $x>x_{1}$. The continuous function $g_{k}^{\prime}$ restricted to the compact set [0, $\left.x_{1}\right]$ is bounded by a real constant $L_{1}>0$. So it follows that $\left|g_{k}^{\prime}(x)\right|<\max \left\{L_{1}, 1+|L|\right\}$ for $x \in[0, \infty)$. Thus, the function $h_{k}=\varepsilon g_{k}$ satisfies the hypothesis (H3) and since it also fulfils the requirements (H1) and (H2) (see the two properties (P1) and (P2) of $g_{k}$ ), we conclude that $\mathscr{M}_{\varepsilon} \subset \mathscr{M}$ for $\varepsilon>0$ sufficiently small and, therefore, $\mathscr{M} \neq \varnothing$. 


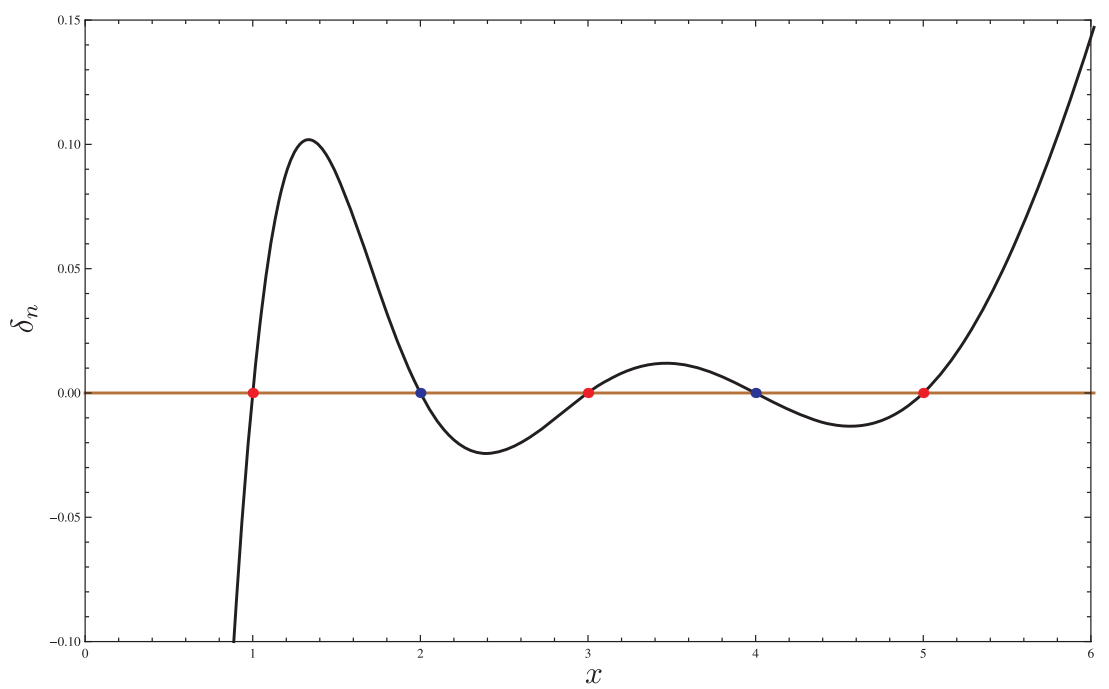

FIG. 4. The continuous black line is the graph of the displacement function associated with (3.2). The stable limit cycles are illustrated by blue dots and the unstable by red dots. We choose $\alpha=2, \beta=1, \gamma=1, n=5, x_{j}=j$, for $j=1, \ldots, 5$ and $\varepsilon=0.08$ in (3.6). (For interpretation of the references to colour in this figure legend, the reader is referred to the web version of this article.)

Two easy examples are obtained choosing the following families of functions

$$
\begin{gathered}
x \in \mathbb{R} \mapsto g_{k}(x)=x^{\alpha} \mathrm{e}^{-\gamma x^{\beta}}\left(\prod_{j=1}^{k}\left(x-x_{j}\right)\right) u(x), \\
x \in \mathbb{R} \mapsto g_{\infty}(x)=x^{\alpha} \mathrm{e}^{-\gamma x^{\beta}} \sin (\omega x) u(x),
\end{gathered}
$$

where $\alpha \geq 2$ and $\beta \geq 1$ are positive integer numbers, $\gamma$ and $\omega$ are positive real numbers, $\left\{x_{1}, \ldots, x_{k}\right\}$ is a finite sequence of positive real numbers and $u: \mathbb{R} \rightarrow \mathbb{R}$ is the Heaviside function, that is, $u(x)=0$, if $x<0$ and $u(x)=1$, if $x \geq 0$.

Figure 4 illustrates the graph of the displacement function of (3.2), when we choose $\alpha=2, \beta=1$, $\gamma=1, n=5, x_{j}=j$, for $j=1, \ldots, 5$, and $\varepsilon=0.08$ in (3.6).

The graph of the displacement function of (3.2), considering now (3.7), is shown in Fig. 5. The parameters used are $\alpha=2, \beta=1, \omega=\pi$ and $\varepsilon=0.01$.

\section{The 3D case}

In this section, we analyse bifurcations of limit cycles from the degenerated T-singularity given by (1.3) when we perturb the separation plane. We stress that the dynamical behaviour of system (1.3) was studied in Carvalho \& Teixeira (2015) and in Subsection 4.1 we just summarize them. In Subsection 4.2, we use Theorem 1.1 in order to prove Theorem 1.2. 


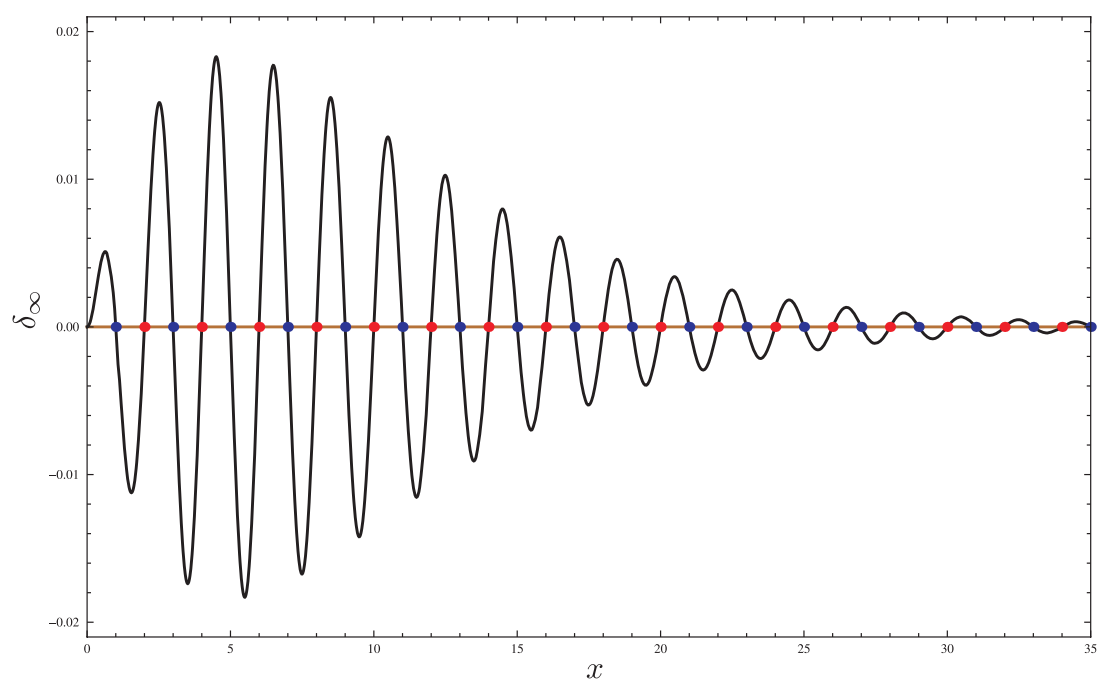

FIG. 5. The continuous black line is the graph of the displacement function associated with (3.2). The stable limit cycles are illustrated by blue dots and the unstable by red dots. We take (3.7) with parameters $\alpha=2, \beta=1, \omega=\pi$ and $\varepsilon=0.01$. (For interpretation of the references to colour in this figure legend, the reader is referred to the web version of this article.)

\subsection{Properties of the unperturbed system}

As stated in Carvalho \& Teixeira (2015), the PWLVF $Z=\left(F^{-}, F^{+}, \mathscr{L}\right)$ given by (1.3) presents the following properties:

1. The axes $x$ and $y$ divide the separation boundary $\Sigma=\mathscr{L}^{-1}(0)=\left\{(x, y, 0) \in \mathbb{R}^{3}\right\}$ into four sets:

$$
\begin{aligned}
\Sigma^{c+}=\left\{(x, y, 0) \in \mathbb{R}^{3} \mid x>0, y<0\right\}, & \Sigma^{c-}=\left\{(x, y, 0) \in \mathbb{R}^{3} \mid x<0, y>0\right\}, \\
\Sigma^{e}=\left\{(x, y, 0) \in \mathbb{R}^{3} \mid x<0, y<0\right\}, & \Sigma^{s}=\left\{(x, y, 0) \in \mathbb{R}^{3} \mid x>0, y>0\right\} .
\end{aligned}
$$

2. The normalized sliding vector field $Z^{s}(x, y, z)=((-1-(x+y)) x+y,(1-(x+y)) x-y, 0)$ given in (2.2) has a saddle-node at the origin (see Fig. 6(a)). As a consequence, the sliding vector field associated to $Z$ has the phase portrait shown in Fig. 6(b). In fact, applying the change of variables $(u, v)=(x+y, x-y)$ on $Z^{s}$ we obtain that its two first coordinates are given by $(\dot{u}, \dot{v})=(-(u+v) u,-2 v)$, which is a canonical form of a planar saddle-node. The change of orientation on $\Sigma^{e}$ follows from Remark 2.1.

3. The plane $\pi_{0}=\left\{(x, y, z) \in \mathbb{R}^{3} \mid x+y=0\right\}$ is $Z$-invariant. Moreover, $\left.Z\right|_{\pi_{0}}$ is a non-degenerate $\Sigma$-centre. In fact, when $x=-y$, given a point $\left(x_{0},-x_{0}, 0\right) \in \Sigma \cap \pi_{0}$, the half return map associated to $F^{-}$(respectively, $\left.F^{+}\right)$is given by $\varphi_{F^{-}}\left(x_{0},-x_{0}, 0\right)=\left(-x_{0}, x_{0}, 0\right)$ (respectively, $\varphi_{F^{+}}\left(x_{0},-x_{0}, 0\right)=$ $\left.\left(-x_{0}, x_{0}, 0\right)\right)$.

4. All the trajectories of $Z$ tend to the plane $\pi_{0}$. Next we elucidate how this convergence occurs. 
(a)

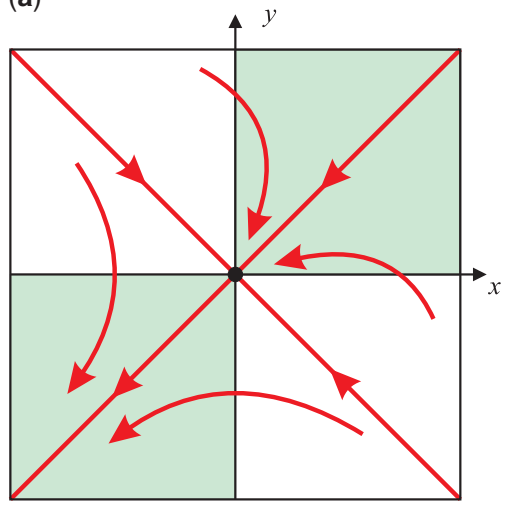

(b)

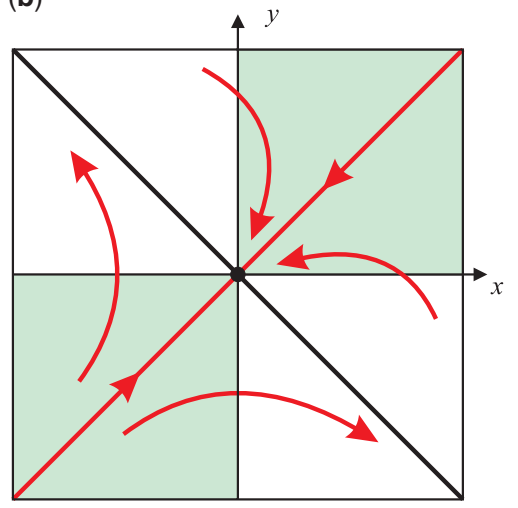

FIG. 6. (a) Phase portrait of the normalized sliding vector field $Z^{s}$. (b) Phase portrait of the sliding vector field associated to $Z$. By (2.1), the sliding vector field vector field $\widehat{Z}^{s}$ is not defined in the line $y=-x$ which is represented by the black line. (For interpretation of the references to colour in this figure legend, the reader is referred to the web version of this article.)

The motivation behind taking the particular canonical form (1.3) in order to represent a T-singularity comes from Colombo \& Jeffrey (2009), where the authors prove that the PWLVF $W=-Z_{0}$, with

$$
Z_{0}(x, y, z)=\left\{\begin{array}{l}
F_{0}^{-}(x, y, z)=(1,-1, x), \quad z \leq 0, \\
F_{0}^{+}(x, y, z)=(-1,1,-y), z \geq 0,
\end{array}\right.
$$

presents a T-singularity with structurally unstable behaviour. In fact, (4.1) has a $\Sigma$-centre at the plane $y=-x$ and all planes $y=-x+k$ are $Z_{0}$-invariant. Of course, small perturbations of (4.1) can destroy the invariance of these sets. In order to perturb the system and get the plane $y=-x$ invariant, an approach like in the centre manifold theorem can be applied. In this case, it is important that all the trajectories of the system converge to the $\Sigma$-centre placed at the plane $y=-x$. In fact, this will ensure that the periodic orbits obtained below are isolated, that is are limit cycles.

As all the planes $y=-x+k$ are invariant by the flow of $Z_{0}$ given by (4.1), in order to construct the PWLVF $Z$ given by (1.3) we take $Z=Z_{0}+\bar{Z}$ where

$$
\bar{Z}(x, y, z)= \begin{cases}\bar{F}^{-}(x, y, z)=(0,0,0), & z \leq 0, \\ \bar{F}^{+}(x, y, z)=(-(x+y),-(x+y), 0), & z \geq 0 .\end{cases}
$$

The phase portrait of (4.2) can be easily pictured and we see that all trajectories of $\bar{Z}$ in $\Sigma^{+}$converge to the plane $y=-x$. So $Z=Z_{0}+\bar{Z}$ keeps the $\Sigma$-centre at $y=-x$ invariant and now, in some sense, the trajectories outside the plane $y=-x$ are globally attracted by the $\Sigma$-centre.

The next step is to apply some monitored perturbation in (1.3) in order to produce the same behaviour as observed by the perturbations of the $\Sigma$-centre described in Section 3 .

\subsection{Proof of Theorem 1.2}

Two PWLVF $Z=\left(F^{-}, F^{+}, \mathscr{H}\right)$ and $\widetilde{Z}=\left(\widetilde{F}^{-}, \widetilde{F}^{+}, \mathscr{H}\right)$ are $\Sigma$-equivalent if there exists an orientation preserving homeomorphism $h$ that sends $\mathscr{H}^{-1}(0)$ to $\mathscr{H}^{-1}(0)$, the orbits of $F^{-}$restricted to $\Sigma^{-}$to the orbits of $\widetilde{F}^{-}$restricted to $\Sigma^{-}$, and the orbits of $F^{+}$restricted to $\Sigma^{+}$to the orbits of $\widetilde{F}^{+}$restricted to $\Sigma^{+}$. 
Note that the restriction of (1.3) to the plane $x+y=0$ is $\Sigma$-equivalent to (1.2). In fact, it is proved in Proposition 6 of Buzzi et al. (2014). So, once we have proved Theorem 1.1, in order to prove Theorem 1.2 it is enough to find a suitable perturbation of (1.3) preserving (H1), (H2) and (H3).

Let $\mathscr{N}$ be the class of all functions $\mathscr{L}_{k}: \mathbb{R}^{3} \rightarrow \mathbb{R}$ defined by

$$
\mathscr{L}_{k}(x, y, z)=z-l_{k}(x, y)
$$

where $l_{k}$ satisfies the following assumptions:

(H1') $l_{k} \in \mathscr{C}^{r}\left(\mathbb{R}^{3}, \mathbb{R}\right)$, with $r \in \mathbb{Z}, r \geq 1$ or $r=\infty$.

(H2') $l_{k}(x, y)=0$, if either $x \leq 0$ or $y \geq 0$ and has a finite $(k \in \mathbb{Z}, k \geq 1)$ or countably infinite $(k=\infty)$ number of zeros when $x=-y>0$. Moreover, if $x_{0}=-y_{0}>0$ is a zero, then

$$
\frac{\partial l_{k}}{\partial(1,-1)}\left(x_{0},-x_{0}\right) \neq 0 \text {. }
$$

Here the previous notation is the derivative of $l_{k}$ on the direction of the vector $(1,-1)$.

(H3') The new separation boundary $\Sigma_{k}=\mathscr{L}_{k}^{-1}(0)$ preserves the partition exhibited by $\Sigma=\mathscr{L}^{-1}(0)$, that is $F^{+} \mathscr{L}_{k}(x, y, z)>0$ and $F^{-} \mathscr{L}_{k}(x, y, z)>0$ when $x>0$ and $y<0$.

Since all the trajectories of $Z=\left(F^{-}, F^{+}, \mathscr{L}\right)$ tend to the plane $x+y=0$, the same holds for $Z_{k}=$ $\left(F^{-}, F^{+}, \mathscr{L}_{k}\right)$ once the sliding and escaping sets remain unchanged and the perturbation $\mathscr{L}_{k}$ of the separation boundary does not modify the smooth vector fields $F^{-}$and $F^{+}$. Moreover, the restriction of $l_{k}$ to the plane $x+y=0$ satisfies (H1), (H2) and (H3) of Section 3. As a consequence $Z_{k}=\left(F^{-}, F^{+}, \mathscr{L}_{k}\right)$ restricted to the plane $x+y=0$ has $k$ hyperbolic limit cycles. The same holds for $k=\infty$. In short, Theorem 1.2 is proved.

\subsection{Some examples}

Let $\phi: \mathbb{R} \rightarrow \mathbb{R}$ be a function such that $\phi(u)=0$, if $u \leq 0, \phi(u)=1$, if $u \geq 1, \phi^{\prime}(u)>0$ for $u \in(0,1)$ and $\phi \in \mathscr{C}^{r}(\mathbb{R}, \mathbb{R}), r \geq 1$ or $r=\infty$. We choose the function $l_{k}: \mathbb{R}^{2} \rightarrow \mathbb{R}$ as

$$
l_{k}(x, y)=\frac{1}{2} h_{k}\left(\frac{x-y}{2}\right) \phi_{\delta}(x) \phi_{\delta}(-y),
$$

where $h_{k}$ has the properties (H1) and $(\mathrm{H} 2)$ and $\phi_{\delta}$ is defined by $\phi_{\delta}(u)=\phi(u / \delta)$, for $\delta>0$. So, $l_{k}$ satisfies (H1') and (H2'). In order to check H3', when $x<0$ or $y>0$

$$
F^{-} \mathscr{L}_{k}(x, y, z)=x, \quad F^{+} \mathscr{L}_{k}(x, y, z)=-y
$$

Analysing the signs of these expressions, we conclude that the sets

$$
\begin{gathered}
\left\{(x, y, 0) \in \mathbb{R}^{3} \mid x>0, y>0\right\}, \\
\left\{(x, y, 0) \in \mathbb{R}^{3} \mid x<0, y>0\right\}, \\
\left\{(x, y, 0) \in \mathbb{R}^{3} \mid x<0, y<0\right\}
\end{gathered}
$$


are, respectively, sliding, crossing and escaping sets. It remains to be seen whether the set $\{(x, y, z) \in$ $\left.\mathbb{R}^{3} \mid x>0, y<0, z=l_{k}(x, y)\right\}$ is a crossing set.

For $x \in(0, \delta)$ and $y \in(-\delta, 0)$, with $\delta>0$ sufficiently small,

$$
\begin{aligned}
F^{-} \mathscr{L}_{k}(x, y, z)= & x-\frac{1}{2} h_{k}\left(\frac{x-y}{2}\right) \phi_{\delta}^{\prime}(x) \phi_{\delta}(-y)- \\
& \frac{1}{2} \phi_{\delta}(x)\left(h_{k}^{\prime}\left(\frac{x-y}{2}\right) \phi_{\delta}(-y)+h_{k}\left(\frac{x-y}{2}\right) \phi_{\delta}^{\prime}(-y)\right), \\
F^{+} \mathscr{L}_{k}(x, y, z)= & -y+\frac{1}{2}(x+y+1) h_{k}\left(\frac{x-y}{2}\right) \phi_{\delta}^{\prime}(x) \phi_{\delta}(-y)+ \\
& \frac{1}{2} \phi_{\delta}(x)\left(h_{k}^{\prime}\left(\frac{x-y}{2}\right) \phi_{\delta}(-y)-(x+y-1) h_{k}\left(\frac{x-y}{2}\right) \phi_{\delta}^{\prime}(-y)\right) .
\end{aligned}
$$

Using the notations $C^{-}(x, y)=F^{-} \mathscr{L}_{k}(x, y, z), C^{+}(x, y)=F^{+} \mathscr{L}_{k}(x, y, z)$, with $z=l_{k}(x, y)$, and $\phi_{\delta}^{(m)}(0)=0$, for $1 \leq m \leq r$, we conclude that the Taylor expansions of $C^{-}$and $C^{+}$at points on the axes $x$ and $y$ are given by

$$
\begin{aligned}
C^{-}(x, y) & =C^{-}(0, y)+\sum_{k=1}^{r-1} \frac{\partial^{k}}{\partial x^{k}} C^{-}(0, y) x^{k}+R_{x}^{-}\left(\|(x, y)\|^{r}\right) \\
& =x+R_{x}^{-}\left(\|(x, y)\|^{r}\right), \\
C^{-}(x, y) & =C^{-}(x, 0)+\sum_{k=1}^{r-1} \frac{\partial^{k}}{\partial x^{k}} C^{-}(x, 0) y^{k}+R_{y}^{-}\left(\|(x, y)\|^{r}\right) \\
& =x+R_{y}^{-}\left(\|(x, y)\|^{r}\right), \\
C^{+}(x, y) & =C^{+}(0, y)+\sum_{k=1}^{r-1} \frac{\partial^{k}}{\partial x^{k}} C^{+}(0, y) x^{k}+R_{x}^{+}\left(\|(x, y)\|^{r}\right) \\
& =-y+R_{x}^{+}\left(\|(x, y)\|^{r}\right), \\
C^{+}(x, y) & =C^{+}(x, 0)+\sum_{k=1}^{r-1} \frac{\partial^{k}}{\partial x^{k}} C^{+}(x, 0) y^{k}+R_{y}^{+}\left(\|(x, y)\|^{r}\right) \\
& =-y+R_{y}^{+}\left(\|(x, y)\|^{r}\right) .
\end{aligned}
$$

So, choosing $\delta>0$ sufficiently small, the set $\left\{(x, y, z) \in \mathbb{R}^{3} \mid x \in(0, \delta), y \in(-\delta, 0), z=l_{k}(x, y)\right\}$ is in fact a crossing set. But this is also true for $x>\delta$ and $y<-\delta$, since

$$
2 y<h_{k}^{\prime}\left(\frac{x-y}{2}\right)<2 x
$$

implies that

$$
F^{-} \mathscr{L}_{k}(x, y, z)=x-\frac{1}{2} h_{k}^{\prime}\left(\frac{x-y}{2}\right)>0, \quad F^{+} \mathscr{L}_{k}(x, y, z)=-y+\frac{1}{2} h_{k}^{\prime}\left(\frac{x-y}{2}\right)>0 .
$$


Restricted to the invariant plane $\left\{(x, y, z) \in \mathbb{R}^{3} \mid x+y=0\right\}$, the condition (4.4) coincides with $\mathbf{H 3}$ and the dynamics of $Z_{k}=\left(F^{-}, F^{+}, \mathscr{L}_{k}\right)$ is given by

$$
\left(x^{\prime}, z^{\prime}\right)=\left\{\begin{array}{l}
(1, x), \quad z \leq \frac{1}{2} h_{k}(x), \\
(-1, x), z \geq \frac{1}{2} h_{k}(x) .
\end{array}\right.
$$

One example of function $l_{k}$ built from (3.6) is given by

$$
\begin{aligned}
l_{k}(x, y) & =\frac{1}{2} \varepsilon g_{k}\left(\frac{x-y}{2}\right) \phi_{\delta}(x) \phi_{\delta}(-y) \\
& =\frac{1}{2} \varepsilon\left(\frac{x-y}{2}\right)^{\alpha} \mathrm{e}^{-\gamma\left(\frac{x-y}{2}\right)^{\beta}}\left(\prod_{j=1}^{k}\left(\frac{x-y}{2}-x_{j}\right)\right) \phi_{\delta}(x) \phi_{\delta}(-y),
\end{aligned}
$$

with $\alpha=2, \beta=1, \gamma=1, n=5, x_{j}=j \in\{1, \ldots, 5\}, \varepsilon=0.08, \phi \in \mathscr{C}^{2}(\mathbb{R}, \mathbb{R})$,

$$
\phi(u)= \begin{cases}0, & u \leq 0, \\ u^{3}\left(6 u^{2}-15 u+10\right), & 0<u<1, \\ 1, & u \geq 1\end{cases}
$$

and $\delta=0.1$.

\section{Funding}

FAPESP (2014/02134-7 to T.C.); CNPq (443302/2014-6 to T.C.); CAPES (1576689 to T.C., from the program PNPD); CAPES CSF-PVE (88881.030454/2013-01 to D.C.B., T.C., L.F.M.); FAPEMIG (PPM-00516-15) and Capes/Estágio Sênior no Exterior number 88881.119020/2016-01 (L.F.M.).

\section{REFERENCES}

Andronov, A., VitT, A. \& KhaikIn, S. (1966) Theory of Oscillators. UK: Pergamon Press Inc.

BRAGA, D. C. \& MelLo, L. F. (2013) Limit cycles in a family of discontinuous piecewise linear differential systems with two zones in the plane. Nonlinear Dynam., 73, 1283-1288.

Braga, D. C. \& Mello, L. F. (2014) More than three limit cycles in discontinuous piecewise linear differential systems with two zones in the plane. Internat. J. Bifur. Chaos Appl. Sci. Engrg., 24, 1450056 (10 pages).

BRAGA, D. C. \& Mello, L. F. (2015) Arbitrary number of limit cycles for planar discontinuous piecewise linear differential systems with two zones. Electron. J. Differential Equations, 2015, 1-12.

Buzzi, C. A., Carvalho T. \& Teixeira, M. A. (2014) Birth of limit cycles bifurcating from a nonsmooth center. J. Math. Pures Appl., 102, 36-47.

Carvalho, T. \& Teixeira, M. A. (2015) Attractivity, degeneracy and codimension of a typical singularity in 3D piecewise smooth vector fields. Preprint, http://arxiv.org/abs/1508.00456.

Colombo, A. \& JefFrey, M. R. (2009) The two-fold singularity of discontinuous vector fields. SIAM J. Appl. Dyn. Syst., 8, 624-640.

COLOMBo, A. \& JefFrey, M. R. (2011) Nondeterministic chaos, and the two-fold singularity in piecewise smooth flows. SIAM J. Appl. Dyn. Syst., 10, 423-451. 
di Bernardo, M., Colombo, A., Fossas, E. \& JefFrey, M. R. (2010) Teixeira singularities in 3D switched feedback control systems. Systems Control Lett., 59, 615-622.

FILIPPOV, A. F. (1988) Differential Equations with Discontinuous Righthand Sides, Mathematics and its Applications (Soviet Series). Dordrecht: Kluwer Academic Publishers.

Freire, E., Ponce, E. \& Torres, F. (2014) A general mechanism to generate three limit cycles in planar Filippov systems with two zones. Nonlinear Dynam., 78, 251-263.

GinouX, J.-M. \& PetitgiraRd, L. (2010) Poincaré's forgotten conferences on wireless telegraphy. Internat. J. Bifur. Chaos Appl. Sci. Engrg., 20, 3617-3626.

Hilbert, D. (1902) Mathematische Probleme, Lecture, Second Internat. Congr. Math. Paris, (1900), Nachr. Ges. Wiss. Göttingen Math. Phys. KL. (1900), 253-297; English transl. Bull. Amer. Math. Soc., 8, 437-479.

Jacquemard, A., Teixeira, M. A. \& Tonon, D. J. (2012) Piecewise smooth reversible dynamical systems at a two-fold singularity. Internat. J. Bifur. Chaos Appl. Sci. Engrg., 22, 1250192 (13 pages).

JACQuemard, A., TeIXeIRA, M. A. \& TonOn, D. J. (2013) Stability conditions in piecewise smooth dynamical systems at a two-fold singularity. J. Dyn. Control Syst., 19, 47-67.

Llibre, J. \& Ponce, E. (2012) Three nested limit cycles in discontinuous piecewise linear differential systems with two zones. Dyn. Contin. Discrete Impuls. Syst. Ser. B Appl. Algorithms, 19, 325-335.

Llibre, J. \& Teruel, A. E. (2014) Introduction to the Qualitative Theory of Differential Systems: Planar, Symmetric and Continuous Piecewise Linear Systems. Basel: Birkhäuser Advanced Texts Basler Lehrbücher, Springer, Birkhäuser/Springer.

TeiXeIRA, M. A. (1990) Stability conditions for discontinuous vector fields. J. Differential Equations, 88, 15-29.

TeIXEIRA, M. A. (2009) Perturbation theory for non-smooth systems. Encyclopedia of Complexity and Systems Science (R. Meyers ed.), vol. 22 (Perturbation Theory). New York: Springer, pp. 6697-6719. 